\title{
Common fixed points via implicit contractions on b-metric-like spaces
}

\author{
Hassen Aydia,b,*, Abdelbasset Felhic ${ }^{\mathrm{c}}$, Slah Sahmim ${ }^{\mathrm{c}}$ \\ ${ }^{a}$ Department of Mathematics, College of Education of Jubail, Imam Abdulrahman Alfaisal University, P. O: 12020, Industrial Jubail \\ 31961, Saudi Arabia. \\ ${ }^{b}$ Department of Medical Research, China Medical University Hospital, China Medical University, Taichung, Taiwan. \\ ${ }^{c}$ Department of Mathematics and Statistics, College of Sciences, King Faisal University, Hafouf, Saudi Arabia.
}

Communicated by Y. J. Cho

\begin{abstract}
In this paper, we introduce some generalized nonlinear contractions via implicit functions and $\alpha$-admissible pair of mappings. We also provide some common fixed point results for above contractions in the class of b-metric-like spaces. We will derive some consequences and corollaries from our obtained results. Some illustrated examples are presented to make effective the concepts and results. (C)2017 All rights reserved.
\end{abstract}

Keywords: Common fixed point, implicit contraction, b-metric-like space.

2010 MSC: 47H10, 54H25, 46J10.

\section{Introduction and preliminaries}

In 2013, the concept of a b-metric-like space was introduced first by Alghamdi et al. [2] as a generalization of a metric-like space $[3,7,17,18,23]$, a b-metric space $[9,16,31,34,35]$ and a partial metric space $[1,6,15,25,30,32,33]$. For some fixed point results on b-metric-like spaces, see [19] and [13].

The definition of a b-metric-like space is given as follows:

Definition 1.1. Let $X$ be non-empty and $s \geqslant 1$. Let $\sigma: X \times X \rightarrow[0, \infty)$ be a function which satisfies:

(d1) $\sigma(x, y)=0$ implies that $x=y$;

(d2) $\sigma(x, y)=\sigma(y, x)$;

(d3) $\sigma(x, y) \leqslant s[\sigma(x, z)+\sigma(z, y)]$.

Then $\sigma$ is called a b-metric-like and the pair $(X, \sigma)$ is called a b-metric-like space.

Definition 1.2. A sequence $\left\{x_{n}\right\}$ converges to $x \in X$ in the b-metric-like space $(X, \sigma)$ if and only if

$$
\lim _{n \rightarrow \infty} \sigma\left(x_{n}, x\right)=\sigma(x, x) .
$$

\footnotetext{
*Corresponding author

Email addresses: hmaydi@uod.edu.sa (Hassen Aydi), afelhi@kfu.edu.sa (Abdelbasset Felhi), ssahmim@kfu.edu.sa (Slah Sahmim)

doi:10.22436/jnsa.010.04.20
}

Received 2016-02-27 
Remark 1.3. In a b-metric-like space, the limit for a convergent sequence is not unique in general.

Definition 1.4. A sequence $\left\{x_{n}\right\}$ is Cauchy in the b-metric-like space $(X, \sigma)$ if and only if $\lim _{n, m \rightarrow \infty} d\left(x_{n}, x_{m}\right)$ exists and is finite.

Definition 1.5. The b-metric-like space $(X, \sigma)$ is complete if and only if each Cauchy sequence in $X$ is convergent.

Lemma 1.6. Let $(X, \sigma)$ be a b-metric-like space and $\left\{x_{n}\right\}$ be a sequence that converges to $u$ with $\sigma(u, u)=0$. Then for each $y, z \in X$

$$
\frac{1}{s} \sigma(u, z) \leqslant \liminf _{n \rightarrow \infty} \sigma\left(x_{n}, z\right) \leqslant s \sigma(u, z) \text { and } \sigma(z, z) \leqslant 2 s \sigma(z, y) .
$$

Definition 1.7. Let $(X, \sigma)$ be a b-metric-like space. The map $T: X \rightarrow X$ is continuous at $u \in X$ if for all $\varepsilon>0$, there exists $\delta>0$ such that

$$
\mathrm{T}\left(\mathrm{B}_{\sigma}(\mathrm{u}, \delta) \subseteq \mathrm{B}_{\sigma}(\mathrm{Tu}, \varepsilon) .\right.
$$

Definition 1.8. Let $(X, \sigma)$ be a b-metric-like space and $T: X \rightarrow X$ be a given mapping. $T$ is said sequentially continuous at $u \in X$ if for each sequence $\left\{x_{n}\right\}$ in $X$ converging to $u$, we have $T x_{n} \rightarrow T u$, that is,

$$
\lim _{n \rightarrow \infty} \sigma\left(T x_{n}, T u\right)=\sigma(T u, T u) .
$$

$T$ is said sequentially continuous on $X$ if $T$ is sequentially continuous at each $u \in X$.

A simple consequence of above two definitions is stated as follows.

Lemma 1.9. Let $(X, \sigma)$ be a b-metric-like space and $\mathrm{T}: \mathrm{X} \rightarrow \mathrm{X}$ be a given mapping. If $\mathrm{T}$ is continuous on $\mathrm{X}$, then it is sequentially continuous on $\mathrm{X}$.

On the other hand, instead of the standard explicit contractive conditions, Popa [28] used the concept of an implicit function in the contractive condition. This direction was the source of several (common) fixed point and coincidence point theorems in various ambient spaces. For more details, see [5, 10-12, 20].

Now, take $s \geqslant 1$ and denote by $\mathbb{N}$ the set of positive integers. Let $\Psi_{s}$ be the set of functions $\psi$ : $[0, \infty) \rightarrow[0, \infty)$ satisfying:

$\left(\psi_{1}\right) \psi$ is nondecreasing,

$\left(\psi_{2}\right) \sum_{n=1}^{\infty} s^{n} \psi^{n}(t)<\infty$ for each $t \in \mathbb{R}^{+}$where $\psi^{n}$ is the $n$th iterate of $\psi$.

Remark 1.10. It is easy to see that if $\psi \in \Psi_{s}$, then $\psi(t)<t$ for any $t>0$ and $\psi(0)=0$.

We introduce the following.

Definition 1.11. Consider $s \geqslant 1$. Let $\Gamma_{s}$ be the set of functions $F\left(t_{1}, \ldots, t_{6}\right): \mathbb{R}_{+}^{6} \rightarrow \mathbb{R}$ such that

(F1) $F$ is nondecreasing in variable $t_{1}$ and nonincreasing in variables $t_{5}$ and $t_{6}$;

(F2) there exists $h_{1} \in \Psi_{s}$ such that for all $u, v \geqslant 0, F(u, v, v, u, s[u+v], 2 s u) \leqslant 0$ implies $u \leqslant h_{1}(v)$, and $F(u, v, u, v, 2 s u, s[u+v]) \leqslant 0$ implies $u \leqslant h_{1}(v)$.

We present the following examples.

Example 1.12. $F\left(t_{1}, \ldots, t_{6}\right)=t_{1}-a t_{2}-b\left(t_{3}+t_{4}\right)-c\left(t_{5}+t_{6}\right)$, where $a, b, c \geqslant 0$ such that $a s+b(1+s)+$ $\operatorname{cs}(s+3)<1$.

Example 1.13. $F\left(t_{1}, \ldots, t_{6}\right)=t_{1}-k \max \left\{t_{2}, \ldots, t_{6}\right\}$, where $k \in\left[0, \frac{1}{s+s^{2}}\right)$.

In 2012, Samet et al. [29] introduced a new useful concept, named as $\alpha$-admissible maps. 
Definition 1.14 ([29]). For a nonempty set $X$, let $T: X \rightarrow X$ and $\alpha: X \times X \rightarrow[0, \infty)$ be given mappings. We say that $T$ is $\alpha$-admissible if for all $x, y \in X$, we have

$$
\alpha(x, y) \geqslant 1 \Longrightarrow \alpha(\mathrm{T} x, \mathrm{~T} y) \geqslant 1
$$

The above concept opens the door to prove many (common) fixed point results, for example see $[4,21,22,24,26,27]$.

Very recently, Aydi [8] generalized Definition 1.14 and introduced the following.

Definition 1.15. For a nonempty set $X$, let $A, B: X \rightarrow X$ and $\alpha: X \times X \rightarrow[0, \infty)$ be mappings. We say that $(A, B)$ is a generalized $\alpha$-admissible pair if for all $x, y \in X$, we have

$$
\alpha(x, y) \geqslant 1 \Longrightarrow \alpha(A x, B y) \geqslant 1 \text { and } \alpha(B y, A x) \geqslant 1
$$

We can rewrite (1.1) as

$$
\alpha(x, y) \geqslant 1 \Longrightarrow \min (\alpha(A x, B y), \alpha(B y, A x)) \geqslant 1
$$

Now, we introduce the concept of $\alpha$-implicit contractive pair of mappings in the setting of b-metric-like spaces.

Definition 1.16. Let $(X, \sigma)$ be a $b$-metric-like space and $A, B: X \rightarrow X$ be given mappings. We say that $(A, B)$ is an $\alpha$-implicit contractive pair of mappings if there exist two functions $\alpha: X \times X \rightarrow[0, \infty)$ and $\mathrm{F} \in \Gamma_{\mathrm{s}}$ such that

$$
F(\alpha(x, y) \sigma(A x, B y), \sigma(x, y), \sigma(x, A x), \sigma(y, B y), \sigma(x, B y), \sigma(y, A x)) \leqslant 0
$$

for all $x, y \in X$.

In this paper, we provide some common fixed point results involving generalized $\alpha$-implicit contractions on b-metric-like spaces. As consequences of our obtained results, we prove some existing fixed point results on $b$-metric spaces and on metric-like spaces. We also provide some examples illustrating our obtained results and the new concepts.

\section{Fixed point theorems}

In this section, we shall state and prove our main results.

Theorem 2.1. Let $(X, \sigma)$ be a complete $\mathrm{b}$-metric-space and $\mathrm{A}, \mathrm{B}: \mathrm{X} \rightarrow \mathrm{X}$ be generalized $\alpha$-implicit contractive pair of mappings. Suppose that

(i) (A, B) is a generalized $\alpha$-admissible pair;

(ii) there exists $x_{0} \in X$ such that $\alpha\left(A x_{0}, x_{0}\right) \geqslant 1$ and $\alpha\left(x_{0}, A x_{0}\right) \geqslant 1$;

(iii) $A$ and $B$ are sequentially continuous on $(X, \sigma)$.

Then there exists $u \in X$ such that

$$
\frac{1}{s} \sigma(u, A u) \leqslant \sigma(A u, A u) \leqslant s \sigma(u, A u), \quad \frac{1}{s} \sigma(u, B u) \leqslant \sigma(B u, B u) \leqslant s \sigma(u, B u), \quad \text { and } \quad \sigma(u, u)=0 .
$$

Assume in addition that

(iv) $\alpha(z, z) \geqslant 1$ for all $z$ verifying (2.1);

(v) F satisfies

$$
\left(F_{\gamma}\right) \text { if } F\left(u, 0, v, w, 2 s^{2} u, 2 s^{2} u\right) \leqslant 0 \text { for all } u, v, w \geqslant 0 \text {, there exists } \gamma \in\left[0, \frac{1}{2 s^{2}}\right) \text { such that } u \leqslant \gamma \max \{v, w\} .
$$


Then $\mathrm{u}$ is a common fixed point of $\mathrm{A}$ and $\mathrm{B}$, that is, $\mathrm{u}=\mathrm{Au}=\mathrm{Bu}$.

Proof. By assumption (ii), there exists a point $x_{0} \in X$ such that $\alpha\left(x_{0}, A x_{0}\right) \geqslant 1$ and $\alpha\left(A x_{0}, x_{0}\right) \geqslant 1$. Take $x_{1}=A x_{0}$ and $x_{2}=B x_{1}$. By induction, we arrive to construct a sequence $\left\{x_{n}\right\}$ such that

$$
\mathrm{x}_{2 \mathrm{n}}=\mathrm{B} \mathrm{x}_{2 \mathrm{n}-1} \quad \text { and } \quad \mathrm{x}_{2 \mathrm{n}+1}=\mathrm{A} \mathrm{x}_{2 \mathrm{n}} \quad \forall \mathrm{n}=1,2, \ldots
$$

We split the proof into several steps:

Step 1: $\alpha\left(x_{n}, x_{n+1}\right) \geqslant 1$ and $\alpha\left(x_{n+1}, x_{n}\right) \geqslant 1$ for all $n=0,1, \ldots$

We have $\alpha\left(x_{0}, x_{1}\right) \geqslant 1$ and $\alpha\left(x_{1}, x_{0}\right) \geqslant 1$. Since $(A, B)$ is a generalized $\alpha$-admissible pair,

$$
\alpha\left(x_{1}, x_{2}\right)=\alpha\left(A x_{0}, B x_{1}\right) \geqslant 1 \text { and } \alpha\left(x_{2}, x_{1}\right)=\alpha\left(B x_{1}, A x_{0}\right) \geqslant 1
$$

We also have

$$
\alpha\left(x_{3}, x_{2}\right)=\alpha\left(A x_{2}, B x_{1}\right) \geqslant 1 \text { and } \alpha\left(x_{2}, x_{3}\right)=\alpha\left(B x_{1}, A x_{2}\right) \geqslant 1
$$

By induction, we obtain

$$
\alpha\left(x_{n}, x_{n+1}\right) \geqslant 1 \text { and } \alpha\left(x_{n+1}, x_{n}\right) \geqslant 1 \text { for all } n=0,1, \ldots
$$

Step 2: $d\left(x_{n}, x_{n+1}\right) \leqslant h_{1}^{n}\left(d\left(x_{0}, x_{1}\right)\right)$ for all $n=0,1, \ldots$

From (1.2), we have

$$
\begin{aligned}
& F\left(\alpha\left(x_{2 n-2}, x_{2 n-1}\right) \sigma\left(A x_{2 n-2}, B x_{2 n-1}\right), \sigma\left(x_{2 n-2}, x_{2 n-1}\right),\right. \\
& \left.\sigma\left(x_{2 n-2}, A x_{2 n-2}\right), \sigma\left(x_{2 n-1}, B x_{2 n-1}\right), \sigma\left(x_{2 n-2}, B x_{2 n-1}\right), \sigma\left(x_{2 n-1}, A x_{2 n-2}\right)\right) \leqslant 0,
\end{aligned}
$$

that is,

$$
\begin{aligned}
& F\left(\alpha\left(x_{2 n-2}, x_{2 n-1}\right) \sigma\left(x_{2 n-1}, x_{2 n}\right), \sigma\left(x_{2 n-2}, x_{2 n-1}\right),\right. \\
& \left.\quad \sigma\left(x_{2 n-2}, x_{2 n-1}\right), \sigma\left(x_{2 n-1}, x_{2 n}\right), \sigma\left(x_{2 n-2}, x_{2 n}\right), \sigma\left(x_{2 n-1}, x_{2 n-1}\right)\right) \leqslant 0 .
\end{aligned}
$$

Using (2.3), (d3) in the fifth variable and (F1) in the first, fifth and sixth variables

$$
\begin{aligned}
& F\left(\sigma\left(x_{2 n-1}, x_{2 n}\right), \sigma\left(x_{2 n-2}, x_{2 n-1}\right), \sigma\left(x_{2 n-2}, x_{2 n-1}\right),\right. \\
& \left.\quad \sigma\left(x_{2 n-1}, x_{2 n}\right), s\left[\sigma\left(x_{2 n-2}, x_{2 n-1}\right)+\sigma\left(x_{2 n-1}, x_{2 n}\right)\right], 2 s \sigma\left(x_{2 n-1}, x_{2 n}\right)\right) \leqslant 0 .
\end{aligned}
$$

By (F2), we obtain

$$
\sigma\left(x_{2 n-1}, x_{2 n}\right) \leqslant h_{1}\left(\sigma\left(x_{2 n-2}, x_{2 n-1}\right)\right)
$$

Similarly, from (1.2), we have

$$
\begin{aligned}
& F\left(\alpha\left(x_{2 n}, x_{2 n-1}\right) \sigma\left(A x_{2 n}, B x_{2 n-1}\right), \sigma\left(x_{2 n}, x_{2 n-1}\right),\right. \\
& \left.\sigma\left(x_{2 n}, A x_{2 n}\right), \sigma\left(x_{2 n-1}, B x_{2 n-1}\right), \sigma\left(x_{2 n}, B x_{2 n-1}\right), \sigma\left(x_{2 n-1}, A x_{2 n}\right)\right) \leqslant 0,
\end{aligned}
$$

that is,

$$
\begin{aligned}
& F\left(\alpha\left(x_{2 n}, x_{2 n-1}\right) \sigma\left(x_{2 n+1}, x_{2 n}\right), \sigma\left(x_{2 n}, x_{2 n-1}\right),\right. \\
& \left.\sigma\left(x_{2 n}, x_{2 n+1}\right), \sigma\left(x_{2 n-1}, x_{2 n}\right), \sigma\left(x_{2 n}, x_{2 n}\right), \sigma\left(x_{2 n-1}, x_{2 n+1}\right)\right) \leqslant 0 .
\end{aligned}
$$

Again, by (2.3), we apply (F1) in the first variable to get

$$
F\left(\sigma\left(x_{2 n+1}, x_{2 n}\right), \sigma\left(x_{2 n}, x_{2 n-1}\right), \sigma\left(x_{2 n}, x_{2 n+1}\right), \sigma\left(x_{2 n-1}, x_{2 n}\right), \sigma\left(x_{2 n}, x_{2 n}\right), \sigma\left(x_{2 n-1}, x_{2 n+1}\right)\right) \leqslant 0 .
$$

Applying again (F1) in the fifth and sixth variables,

$$
\begin{aligned}
& F\left(\sigma\left(x_{2 n+1}, x_{2 n}\right), \sigma\left(x_{2 n}, x_{2 n-1}\right), \sigma\left(x_{2 n}, x_{2 n+1}\right),\right. \\
& \left.\sigma\left(x_{2 n-1}, x_{2 n}\right), 2 s \sigma\left(x_{2 n}, x_{2 n+1}\right), s\left[\sigma\left(x_{2 n-1}, x_{2 n}\right)+\sigma\left(x_{2 n}, x_{2 n+1}\right)\right]\right) \leqslant 0 .
\end{aligned}
$$


By (F2),

$$
\sigma\left(x_{2 n}, x_{2 n+1}\right) \leqslant h_{1}\left(\sigma\left(x_{2 n-1}, x_{2 n}\right)\right) .
$$

Combining (2.4) and (2.5), we obtain

$$
\sigma\left(x_{n}, x_{n+1}\right) \leqslant h_{1}\left(\sigma\left(x_{n-1}, x_{n}\right)\right) \text { for all } n=1,2, \ldots
$$

We deduce

$$
\sigma\left(x_{n}, x_{n+1}\right) \leqslant h_{1}^{n}\left(\sigma\left(x_{0}, x_{1}\right)\right) \text { for all } n=0,1,2, \ldots
$$

Step 3: $\left\{x_{n}\right\}$ is a Cauchy sequence.

Now, we shall prove that $\left\{x_{n}\right\}$ is a Cauchy sequence in the b-metric-like space $(X, \sigma)$.

Using (d3) and (2.6), we have for all $n, k \in \mathbb{N}$

$$
\begin{aligned}
\sigma\left(x_{n}, x_{n+k}\right) & \leqslant s \sigma\left(x_{n}, x_{n+1}\right)+s^{2} \sigma\left(x_{n+1}, x_{n+2}\right)+\ldots+s^{k-1} \sigma\left(x_{n+k-1}, x_{n+k}\right) \\
& \leqslant\left(\operatorname{sh}_{1}^{n}+s^{2} h_{1}^{n+1}+\ldots+s^{k-1} h_{1}^{n+k-1}\right)\left[\sigma\left(x_{0}, x_{1}\right)\right] \\
& \leqslant \sum_{i=n}^{n+k-1} s^{i} h_{1}^{i}\left[\sigma\left(x_{0}, x_{1}\right)\right] \leqslant \sum_{i=n}^{\infty} s^{i} h_{1}^{i}\left[\sigma\left(x_{0}, x_{1}\right)\right] .
\end{aligned}
$$

Since $h_{1} \in \Psi_{s}$,

$$
\sigma\left(x_{n}, x_{n+k}\right) \rightarrow 0 \quad \text { as } n \rightarrow \infty \text {, for all } k .
$$

It follows that $\left\{x_{n}\right\}$ is a Cauchy sequence in the b-metric-like space $(X, \sigma)$.

Step 4: Existence of a common fixed point.

Since $(X, \sigma)$ is complete, the sequence $\left\{x_{n}\right\}$ converges to some $u \in X$, that is,

$$
\sigma(u, u)=\lim _{n \rightarrow \infty} \sigma\left(x_{n}, u\right)=\lim _{m, n \rightarrow \infty} \sigma\left(x_{n}, x_{m}\right)
$$

By (2.7), we get

$$
\sigma(u, u)=\lim _{n \rightarrow \infty} \sigma\left(x_{n}, u\right)=\lim _{m, n \longrightarrow \infty} \sigma\left(x_{n}, x_{m}\right)=0
$$

This implies that

$$
\lim _{n \rightarrow \infty} \sigma\left(x_{2 n+1}, u\right)=\lim _{n \rightarrow \infty} \sigma\left(x_{2 n+2}, u\right)=0
$$

By (2.2),

$$
\lim _{n \rightarrow \infty} \sigma\left(A x_{2 n}, u\right)=\lim _{n \rightarrow \infty} \sigma\left(B x_{2 n+1}, u\right)=0 .
$$

We shall prove that $u=A u=B u$, that is, $u$ is a common fixed point of $A$ and $B$.

Using the sequentially continuity of $A$ and the fact that $x_{2 n} \rightarrow u$ in $(X, \sigma)$,

$$
\lim _{n \rightarrow \infty} \sigma\left(x_{2 n+1}, A u\right)=\lim _{n \rightarrow \infty} \sigma\left(A x_{2 n}, A u\right)=\sigma(A u, A u) .
$$

Using now the sequentially continuity of $B$ and the fact that $x_{2 n+1} \rightarrow u$ in $(X, \sigma)$,

$$
\lim _{n \rightarrow \infty} \sigma\left(x_{2 n+2}, B u\right)=\lim _{n \rightarrow \infty} \sigma\left(B x_{2 n+1}, B u\right)=\sigma(B u, B u) .
$$

On the other side, having $\lim _{n \rightarrow \infty} \sigma\left(x_{n}, u\right)=0=\sigma(u, u)$ together with Lemma 1.6,

$$
\frac{1}{s} \sigma(u, A u) \leqslant \lim _{n \rightarrow \infty} \sigma\left(x_{2 n+1}, A u\right) \leqslant s \sigma(u, A u) .
$$

This yields that

$$
\frac{1}{s} \sigma(u, A u) \leqslant \sigma(A u, A u) \leqslant s \sigma(u, A u) .
$$


Similarly

$$
\frac{1}{\mathrm{~s}} \sigma(\mathrm{u}, \mathrm{Bu}) \leqslant \sigma(\mathrm{Bu}, \mathrm{Bu}) \leqslant s \sigma(\mathrm{u}, \mathrm{Bu}) .
$$

By (2.8), (2.9), and (2.10), the assertion (2.1) holds. So by condition (iv), we have

$$
\alpha(u, u) \geqslant 1
$$

Now, applying (1.2) for $x=y=u$

$$
F(\alpha(u, u) \sigma(A u, B u), \sigma(u, u), \sigma(u, A u), \sigma(u, B u), \sigma(u, B u), \sigma(u, A u)) \leqslant 0,
$$

i.e,

$$
F(\alpha(u, u) \sigma(A u, B u), 0, \sigma(u, A u), \sigma(u, B u), \sigma(u, B u), \sigma(u, A u)) \leqslant 0 .
$$

Since $\alpha(u, u) \geqslant 1$, applying (F1) for the first variable, we get

$$
F(\sigma(A u, B u), 0, \sigma(u, A u), \sigma(u, B u), \sigma(u, B u), \sigma(u, A u)) \leqslant 0 .
$$

Remember that

$$
\sigma(u, B u) \leqslant s \sigma(B u, B u) \leqslant 2 s^{2} \sigma(A u, B u),
$$

so applying (F1) in the fifth and sixth variables, we obtain

$$
F\left(\sigma(A u, B u), 0, \sigma(u, A u), \sigma(u, B u), 2 s^{2} \sigma(A u, B u), 2 s^{2} \sigma(A u, B u)\right) \leqslant 0 .
$$

Recall that $F$ satisfies property $\left(F_{\gamma}\right)$, so there exists $\gamma \in\left[0, \frac{1}{2 s^{2}}\right)$ such that

$$
\sigma(A u, B u) \leqslant \gamma \max \{\sigma(u, A u), \sigma(u, B u)\} \leqslant 2 \gamma s^{2} \sigma(A u, B u),
$$

which holds unless $\sigma(A u, B u)=0$. Thus, $A u=B u$. We deduce from (2.11) that $\sigma(u, A u)=0=\sigma(u, B u)$. Hence

$$
u=A u=B u .
$$

This completes the proof.

If we take $A=B$ in Theorem 2.1, we get the following result.

Corollary 2.2. Let $(\mathrm{X}, \sigma)$ be a complete $\mathrm{b}$-metric-like space and $\mathrm{A}: \mathrm{X} \rightarrow \mathrm{X}$ be a given mapping. Assume there exists $\mathrm{F} \in \Gamma_{\mathrm{s}}$ such that

$$
F(\alpha(x, y) \sigma(A x, A y), \sigma(x, y), \sigma(x, A x), \sigma(y, A y), \sigma(x, A y), \sigma(y, A x)) \leqslant 0
$$

for all $x, y \in X$. Suppose that

(i) $(A, A)$ is an $\alpha$-admissible;

(ii) there exists $x_{0} \in X$ such that $\alpha\left(A x_{0}, x_{0}\right) \geqslant 1$ and $\alpha\left(x_{0}, A x_{0}\right) \geqslant 1$;

(iii) $A$ is sequentially continuous on $(X, \sigma)$.

Then there exists $u \in X$ such that

$$
\frac{1}{s} \sigma(u, A u) \leqslant \sigma(A u, A u) \leqslant s \sigma(u, A u) .
$$

Assume in addition that

(iv) $\alpha(z, z) \geqslant 1$ for all $z$ verifying (2.12);

(v) F satisfies 
$\left(F_{\gamma}\right)$ if $F\left(u, 0, v, w, 2 s^{2} u, 2 s^{2} u\right) \leqslant 0$ for all $u, v, w \geqslant 0$, there exists $\gamma \in\left[0, \frac{1}{2 s^{2}}\right)$ such that $u \leqslant \gamma \max \{v, w\}$. Then $u$ is a fixed point of $A$, that is, $u=A u$.

Proof. The proof follows from the lines in the proof of Theorem 2.1.

Considering the b-metric case in Theorem 2.1, we have the following.

Corollary 2.3. Let $(X, \sigma)$ be a complete $\mathrm{b}$-metric space and $\mathrm{A}, \mathrm{B}: \mathrm{X} \rightarrow \mathrm{X}$ be given mappings. Suppose there exists $\mathrm{F} \in \Gamma_{\mathrm{s}}$ such that

$$
F(\alpha(x, y) \sigma(A x, B y), \sigma(x, y), \sigma(x, A x), \sigma(y, B y), \sigma(x, B y), \sigma(y, A x)) \leqslant 0
$$

for all $\mathrm{x}, \mathrm{y} \in \mathrm{X}$. Assume that

(i) $(A, B)$ is an $\alpha$-admissible pair;

(ii) there exists $x_{0} \in X$ such that $\alpha\left(x_{0}, A x_{0}\right) \geqslant 1$ and $\alpha\left(A x_{0}, x_{0}\right) \geqslant 1$;

(iii) $A$ and $B$ are sequentially continuous on $(X, \sigma)$.

Then there exists $u \in X$ such that $u=A u=B u$.

Proof. Due to conditions (i)-(iii), there exists $u \in X$ such that from Theorem 2.1, (2.1) becomes

$$
\mathrm{u}=\mathrm{A} u=\mathrm{Bu},
$$

that is, $u$ is a common fixed point of $A$ and B. Here, we do not need conditions (iv) and (v) given in Theorem 2.1.

Corollary 2.4. Let $(\mathrm{X}, \sigma)$ be a complete b-metric space and $\mathrm{A}: \mathrm{X} \rightarrow \mathrm{X}$ a given mapping. Assume there exists $\mathrm{F} \in \Gamma_{\mathrm{s}}$ such that

$$
F(\alpha(x, y) \sigma(A x, A y), \sigma(x, y), \sigma(x, A x), \sigma(y, A y), \sigma(x, A y), \sigma(y, A x)) \leqslant 0
$$

for all $x, y \in X$. Suppose that

(i) $(A, A)$ is an $\alpha$-admissible mapping;

(ii) there exists $x_{0} \in X$ such that $\alpha\left(A x_{0}, x_{0}\right) \geqslant 1$ and $\alpha\left(x_{0}, A x_{0}\right) \geqslant 1$;

(iii) $A$ is sequentially continuous on $(X, \sigma)$.

Then there exists $u \in X$ such that $u=A u$.

Now, consider in Theorem 2.1 the operator F presented by Example 1.13. We state the following.

Corollary 2.5. Let $(\mathrm{X}, \sigma)$ be a complete $\mathrm{b}$-metric like metric space and $\mathrm{A}, \mathrm{B}: \mathrm{X} \rightarrow \mathrm{X}$ satisfying

$$
\alpha(x, y) \sigma(A x, B y) \leqslant k \max \{\sigma(x, y), \sigma(x, A x), \sigma(y, B y), \sigma(x, B y), \sigma(y, A x)\},
$$

where $k \in\left[0, \frac{1}{2 s^{2}}\right)$. Suppose that

(i) $(A, B)$ is an $\alpha$-admissible pair;

(ii) there exists $x_{0} \in X$ such that $\alpha\left(x_{0}, A x_{0}\right) \geqslant 1$ and $\alpha\left(A x_{0}, x_{0}\right) \geqslant 1$;

(iii) $A$ and $B$ are sequentially continuous on $(X, \sigma)$.

Then, there exists $\mathrm{u} \in \mathrm{X}$ such that $\mathrm{u}$ is a common fixed point of $\mathrm{A}$ and $\mathrm{B}$, that is, $\mathrm{u}=\mathrm{Au}=\mathrm{Bu}$.

For $A=B$, we have the following result.

Corollary 2.6. Let $(\mathrm{X}, \sigma)$ be a complete b-metric-like space and $\mathrm{A}: \mathrm{X} \rightarrow \mathrm{X}$ be a mapping such that

$$
\alpha(x, y) \sigma(A x, A y) \leqslant k \max \{\sigma(x, y), \sigma(x, A x), \sigma(y, A y), \sigma(x, A y), \sigma(y, A x)\},
$$

where $\mathrm{k} \in\left[0, \frac{1}{2 \mathrm{~s}^{2}}\right)$. Suppose that 
(i) $(\mathrm{A}, \mathrm{A})$ is an $\alpha$-admissible mapping;

(ii) there exists $x_{0} \in X$ such that $\alpha\left(x_{0}, A x_{0}\right) \geqslant 1$ and $\alpha\left(A x_{0}, x_{0}\right) \geqslant 1$;

(iii) $A$ is continuous on $(X, \sigma)$.

Then there exists $u \in X$ such that $u$ is a fixed point of $A$, that is, $u=A u$.

In the next result, we drop the sequentially continuity hypothesis of $A$ and $B$ and we replace it by the following.

(H) If $\left\{x_{n}\right\}$ is a sequence in $X$ such that $\alpha\left(x_{n}, x_{n+1}\right) \geqslant 1$ and $\alpha\left(x_{n+1}, x_{n}\right) \geqslant 1$ for all $n$ and $x_{n} \rightarrow x \in X$ as $n \rightarrow \infty$, then there exists a subsequence $\left\{x_{n(k)}\right\}$ of $\left\{x_{n}\right\}$ such that $\alpha\left(x_{n(k)}, x\right) \geqslant 1$ and $\alpha\left(x, x_{n(k)}\right) \geqslant 1$ for all $k$.

We also replace Definition 1.11 by the following.

Definition 2.7. Consider $s \geqslant 1$. Let $G_{s}$ be the set of continuous functions $F\left(t_{1}, \ldots, t_{6}\right): \mathbb{R}_{+}^{6} \rightarrow \mathbb{R}$ such that

(G1) $F$ is nondecreasing in variable $t_{1}$ and nonincreasing in variables $t_{5}$ and $t_{6}$;

(G2) there exists $h_{1} \in \Psi_{s}$ such that for all $u, v \geqslant 0, F\left(\frac{u}{s}, v, v, u, s[u+v], 2 s u\right) \leqslant 0$ implies $u \leqslant h_{1}(v)$, and $\mathrm{F}\left(\frac{\mathrm{u}}{\mathrm{s}}, v, \mathrm{u}, v, 2 \mathrm{su}, \mathrm{s}[\mathrm{u}+v]\right) \leqslant 0$ implies $u \leqslant h_{1}(v)$.

Remark 2.8. If $\mathrm{F} \in \Gamma_{\mathrm{s}}$, the continuity of $\mathrm{F}$ is not required. But, in Definition 2.7, the continuity is essential. Also, the property (G2) implies (F2). The converse sense is not true. Therefore, the sets $\Gamma_{s}$ and $G_{s}$ are different to each other.

Theorem 2.9. Let $(X, \sigma)$ be a complete $\mathrm{b}$-metric-like space and $\mathrm{A}, \mathrm{B}: \mathrm{X} \rightarrow \mathrm{X}$ be generalized $\alpha$-implicit contractive pair of mappings (with $\mathrm{F} \in \mathrm{G}_{\mathrm{s}}$ ). Suppose that

(i) $(A, B)$ is an $\alpha$-admissible pair;

(ii) there exists $x_{0} \in X$ such that $\alpha\left(x_{0}, A x_{0}\right) \geqslant 1$ and $\alpha\left(A x_{0}, x_{0}\right) \geqslant 1$;

(iii) $(\mathrm{H})$ holds.

Then there exists $\mathrm{u} \in \mathrm{X}$ such that $\mathrm{u}=\mathrm{A} u=\mathrm{Bu}$. We also have $\sigma(\mathrm{u}, \mathrm{u})=0$.

Proof. Following the proof of Theorem 2.1, the sequence $\left\{x_{n}\right\}$ defined by (2.2) is Cauchy and converges to some $u \in X$ in $(X, \sigma)$. Remember that (2.3) holds, so from condition (iv), there exists a subsequence $\left\{x_{n(k)}\right\}$ of $\left\{x_{n}\right\}$ such that $\alpha\left(x_{2 n(k)}, u\right) \geqslant 1$ and $\alpha\left(u, x_{2 n(k)-1}\right) \geqslant 1$ for all $k$. We shall show that $u=A u=B u$.

Taking $x=x_{2 n(k)}$ and $y=u$ in (1.2)

$$
\begin{aligned}
\limsup _{k \rightarrow \infty} F\left(\alpha\left(x_{2 n(k)}, u\right) \sigma\left(A x_{2 n(k)}, B u\right)\right. & \sigma\left(x_{2 n(k)}, u\right), \sigma\left(x_{2 n(k)}, A x_{2 n(k)}\right), \\
& \left.\sigma(u, B u), \sigma\left(x_{2 n(k)}, B u\right), \sigma\left(u, A x_{2 n(k)}\right)\right) \leqslant 0 .
\end{aligned}
$$

Having $\alpha\left(x_{2 n(k)}, u\right) \geqslant 1$, so applying (G1) in the first variable, we have

$$
F\left(\sigma\left(x_{2 n(k)+1}, B u\right), \sigma\left(x_{2 n(k)}, u\right), \sigma\left(x_{2 n(k)}, x_{2 n(k)+1}\right), \sigma(u, B u), \sigma\left(x_{2 n(k)}, B u\right), \sigma\left(u, x_{2 n(k)+1}\right)\right) \leqslant 0 .
$$

We know that $\lim _{n \rightarrow \infty} \sigma\left(x_{n}, u\right)=0=\sigma(u, u)$, so Lemma 1.6 implies that

$$
\frac{1}{s} \sigma(u, B u) \leqslant \limsup _{n \rightarrow \infty} \sigma\left(x_{2 n}(k)+1, B u\right) \leqslant s \sigma(u, B u) .
$$

Letting $k$ to infinity and using continuity of $F$, we have

$$
F\left(\frac{1}{s} \sigma(u, B u), 0,0, \sigma(u, B u), s \sigma(u, B u), 0\right) \leqslant 0 .
$$


Using (G1) in the fifth and sixth variables,

$$
F\left(\frac{1}{s} \sigma(u, B u), 0,0, \sigma(u, B u), s \sigma(u, B u), 2 s \sigma(u, B u)\right) \leqslant 0 .
$$

By (G2), it follows that $p(u, B u) \leqslant h_{1}(0)=0$, which implies that $u=B u$.

Similarly, by taking $x=u$ and $y=x_{2 n(k)-1}$ in (1.2), we have

$$
\begin{gathered}
F\left(\alpha\left(u, x_{2 n(k)-1}\right) \sigma\left(A u, B x_{2 n(k)-1}\right), \sigma\left(u, x_{2 n(k)-1}\right), \sigma(u, A u), \sigma\left(x_{2 n(k)-1}, B x_{2 n(k)-1}\right),\right. \\
\left.\sigma\left(u, B x_{2 n(k)-1}\right), \sigma\left(x_{2 n(k)-1}, A u\right)\right) \leqslant 0 .
\end{gathered}
$$

By $(\mathrm{G} 1)$ and $\alpha\left(u, x_{2 n(k)-1}\right) \geqslant 1$, we get

$$
F\left(\sigma\left(A u, x_{2 n(k)}\right), \sigma\left(u, x_{2 n(k)-1}\right), \sigma(u, A u), \sigma\left(x_{2 n(k)-1}, x_{2 n(k)}\right), \sigma\left(u, x_{2 n(k)}\right), \sigma\left(x_{2 n(k)-1}, A u\right)\right) \leqslant 0 .
$$

Again, in view of Lemma 1.6 and $\lim _{n \rightarrow \infty} \sigma\left(x_{n}(k), u\right)=0=\sigma(u, u)$, we have

$$
\frac{1}{s} \sigma(u, A u) \leqslant \limsup _{n \rightarrow \infty} \sigma\left(x_{2 n(k)}, A u\right) \leqslant s \sigma(u, A u) .
$$

Letting $k \rightarrow \infty$ and using the continuity of $F$, we have

$$
F\left(\frac{1}{s} \sigma(A u, u), 0, \sigma(u, A u), 0,0, s \sigma(u, A u)\right) \leqslant 0 .
$$

Using (G1) in the fifth and sixth variables

$$
\mathrm{F}\left(\frac{1}{\mathrm{~s}} \sigma(A u, u), 0, \sigma(u, A u), 0,2 s \sigma(u, A u), s \sigma(u, A u)\right) \leqslant 0 .
$$

By $(G 2)$, we obtain $\sigma(u, A u) \leqslant h_{1}(0)=0$, that is, $u=A u$.

We state the following corollaries.

Corollary 2.10. Let $(\mathrm{X}, \sigma)$ be a complete $\mathrm{b}$-metric-like space and $\mathrm{A}: \mathrm{X} \rightarrow \mathrm{X}$ a given mapping. Assume there exists $\mathrm{F} \in \mathrm{G}_{\mathrm{s}}$ such that

$$
F(\alpha(x, y) \sigma(A x, A y), \sigma(x, y), \sigma(x, A x), \sigma(y, A y), \sigma(x, A y), \sigma(y, A x)) \leqslant 0
$$

for all $x, y \in X$. Suppose that

(i) $(A, A)$ is an $\alpha$-admissible pair;

(ii) there exists $x_{0} \in X$ such that $\alpha\left(x_{0}, A x_{0}\right) \geqslant 1$ and $\alpha\left(A x_{0}, x_{0}\right) \geqslant 1$;

(iii) $(\mathrm{H})$ is satisfied.

Then there exists $u \in X$ such that $u=A u$. We also have $\sigma(u, u)=0$.

Proof. Taking B = A in Theorem 2.9, we get the above result.

Corollary 2.11. Let $(X, \sigma)$ be a complete $\mathrm{b}$-metric-like space and $\mathrm{A}, \mathrm{B}: \mathrm{X} \rightarrow \mathrm{X}$ satisfying

$$
\alpha(x, y) \sigma(A x, B y) \leqslant k \max \{\sigma(x, y), \sigma(x, A x), \sigma(y, B y), \sigma(x, B y), \sigma(y, A x)\},
$$

where $\mathrm{k} \in\left[0, \frac{1}{\mathrm{~s}^{3}+\mathrm{s}^{2}}\right)$. Suppose that

(i) $(A, B)$ is an $\alpha$-admissible pair;

(ii) there exists $x_{0} \in X$ such that $\alpha\left(A x_{0}, x_{0}\right) \geqslant 1$ and $\alpha\left(x_{0}, A x_{0}\right) \geqslant 1$;

(iii) $(\mathrm{H})$ is satisfied. 
Then there exists $u \in X$ such that $u=A u=B u$.

Proof. It suffices to consider in Theorem 2.9 the operator $F$ given by Example 1.13 with $k \in\left[0, \frac{1}{s^{3}+s^{2}}\right)$. Mention that $F$ is continuous.

Corollary 2.12. Let $(\mathrm{X}, \sigma)$ be a complete b-metric-like space and $\mathrm{A}: \mathrm{X} \rightarrow \mathrm{X}$ be a mapping such that

$$
\alpha(x, y) \sigma(A x, A y) \leqslant k \max \{\sigma(x, y), \sigma(x, A x), \sigma(y, A y), \sigma(x, A y), \sigma(y, A x)\}
$$

for all $x, y \in X$, where $k \in\left[0, \frac{1}{s^{3}+s^{2}}\right)$. Suppose that

(i) $(A, A)$ is an $\alpha$-admissible pair;

(ii) there exists $x_{0} \in X$ such that $\alpha\left(x_{0}, A x_{0}\right) \geqslant 1$ and $\alpha\left(A x_{0}, x_{0}\right) \geqslant 1$;

(iii) $(\mathrm{H})$ is satisfied.

Then there exists $u \in X$ such that $u=A u$.

Proof. Consider in Corollary 2.10 the operator F given by Example 1.13 with $k \in\left[0, \frac{1}{\mathrm{~s}^{3}+\mathrm{s}^{2}}\right)$.

The following two corollaries are Ćirić [14] type results in the setting of b-metric-like spaces.

Corollary 2.13. Let $(X, \sigma)$ be a complete b-metric-like space and $A, B: X \rightarrow X$ satisfying

$$
\sigma(A x, B y) \leqslant k \max \{\sigma(x, y), \sigma(x, A x), \sigma(y, B y), \sigma(x, B y), \sigma(y, A x)\},
$$

where $\mathrm{k} \in\left[0, \frac{1}{\mathrm{~s}^{3}+\mathrm{s}^{2}}\right)$. Then there exists $\mathrm{u} \in \mathrm{X}$ such that $\mathrm{u}=\mathrm{Au}=\mathrm{Bu}$.

Proof. It suffices to take $\alpha(x, y)=1$ in Corollary 2.11 .

Corollary 2.14. Let $(\mathrm{X}, \sigma)$ be a complete $\mathrm{b}$-metric-like space and $\mathrm{A}: \mathrm{X} \rightarrow \mathrm{X}$ be a mapping such that

$$
\sigma(A x, A y) \leqslant k \max \{\sigma(x, y), \sigma(x, A x), \sigma(y, A y), \sigma(x, A y), \sigma(y, A x)\},
$$

where $\mathrm{k} \in\left[0, \frac{1}{\mathrm{~s}^{3}+\mathrm{s}^{2}}\right)$. Then there exists $\mathrm{u} \in \mathrm{X}$ such that $\mathrm{u}=\mathrm{Au}$.

Proof. The proof follows easily when taking $\alpha(x, y)=1$ in Corollary 2.12.

To prove uniqueness of the common fixed point given in Theorem 2.1 (resp. Theorem 2.9), we need to take the following additional hypotheses.

(U) For all $x, y \in \operatorname{CF}(A, B)$, we have $\alpha(x, y) \geqslant 1$, where $\operatorname{CF}(A, B)$ denotes the set of common fixed points of $A$ and $B$, and

(F3) For all $t>0$, we have $F(t, t, 0,0, t, t)>0$.

Theorem 2.15. Adding conditions (U) and (F3) to the hypotheses of Theorem 2.1 (resp. Theorem 2.9), we obtain that $\mathrm{u}$ is the unique common fixed point of $\mathrm{A}$ and $\mathrm{B}$.

Proof. We argue by contradiction, that is, there exist $u, v \in X$ such that $u=A u=B u$ and $v=A v=B v$ with $u \neq v$. By Theorem 2.1 (resp. Theorem 2.9), such $u$ and $v$ satisfy $\sigma(u, u)=\sigma(v, v)=0$. From (1.2), we get

$$
F(\alpha(u, v) \sigma(A u, A v), \sigma(u, v), \sigma(u, A u), \sigma(v, B v), \sigma(u, B v), \sigma(v, A u)) \leqslant 0
$$

i.e,

$$
\mathrm{F}(\alpha(u, v) \sigma(u, v), \sigma(u, v), \sigma(u, u), \sigma(v, v), \sigma(u, v), \sigma(v, u)) \leqslant 0 .
$$

Since $\alpha(u, v) \geqslant 1$, by $(\mathrm{F} 1)=(\mathrm{G} 1)$ in the first variable, we get

$$
F(\sigma(u, v), \sigma(u, v), 0,0, \sigma(u, v), \sigma(u, v)) \leqslant 0 .
$$

Again F satisfies property (F3), so it is a contradiction. Hence $u=v$. 
Theorem 2.16. Adding conditions(U) and (F3) to the hypotheses of Corollary 2.2 (resp. Corollary 2.10), we obtain that $\mathrm{u}$ is the unique fixed point of $\mathrm{A}$.

The following examples illustrate Theorem 2.15 where the considered mappings $A$ and $B$ have a unique common fixed point.

Example 2.17. Take $X=[0, \infty)$ endowed with the complete b-metric-like $\sigma(x, y)=(x+y)^{2}$ with $s=2$. Consider the mappings $A, B: X \rightarrow X$ given by

$$
A x=\left\{\begin{array}{ll}
\frac{x}{9}, & \text { if } x \in[0,1], \\
(x-1)^{2}+\frac{1}{9}, & \text { if } x>1,
\end{array} \text { and } B x= \begin{cases}\frac{x}{9}, & \text { if } x \in[0,1] \\
\frac{2 x^{2}-1}{9}, & \text { if } x>1 .\end{cases}\right.
$$

Define the mapping $\alpha: X \times X \rightarrow[0, \infty)$ by

$$
\alpha(x, y)= \begin{cases}1, & \text { if } x, y \in[0,1] \\ 0, & \text { otherwise }\end{cases}
$$

Let $F\left(t_{1}, t_{2}, t_{3}, t_{4}, t_{5}, t_{6}\right)=t_{1}-\frac{1}{10}\left(t_{5}+t_{6}\right)$. It is obvious that

(i) $(A, B)$ is an $\alpha$-admissible pair;

(ii) there exists $x_{0} \in X$ such that $\alpha\left(x_{0}, A x_{0}\right) \geqslant 1$ and $\alpha\left(A x_{0}, x_{0}\right) \geqslant 1$;

(iii) $\mathrm{F}\left(u, 0, v, w, 2 s^{2} u, 2 s^{2} u\right) \leqslant 0$ for all $u, v, w \geqslant 0$.

Moreover, $A$ and $B$ are sequentially continuous on $(X, \sigma)$. Indeed, let $\left\{x_{n}\right\}$ be a sequence in $X$ such that $x_{n} \rightarrow x$ in $(X, \sigma)$ as $n \rightarrow \infty$. It follows that

$$
\sigma\left(x_{n}, x\right)=\left(x_{n}+x\right)^{2} \rightarrow \sigma(x, x)=4 x^{2} \Leftrightarrow x_{n}+x \rightarrow 2 x \Leftrightarrow\left|x_{n}-x\right| \rightarrow 0 .
$$

$A$ is continuous on $(X,||$.$) , so \left|A x_{n}-A x\right| \rightarrow 0$ as $n \rightarrow \infty$. Then

$$
\sigma\left(A x_{n}, A x\right) \rightarrow \sigma(A x, A x) .
$$

Thus $A$ is sequentially continuous on $(X, \sigma)$. Similarly, B is sequentially continuous on $(X, \sigma)$.

Now, we shall show that $(A, B)$ is an $\alpha$-implicit contractive pair. Let $x, y \in X$ such that $\alpha(x, y)=1$. So, $x, y \in[0,1]$. We have

$$
\begin{aligned}
\mathrm{F}(\alpha(x, y) \sigma(A x, B y), \sigma(x, y), \sigma(x, A x), \sigma(y, B y), \sigma(x, B y), \sigma(y, A x)) & =\sigma(A x, B y)-\frac{1}{9}(\sigma(x, B y)+\sigma(y, A x)) \\
& =\left(\frac{x}{9}+\frac{y}{9}\right)^{2}-\frac{1}{10}\left(\left(x+\frac{y}{9}\right)^{2}+\left(y+\frac{x}{9}\right)^{2}\right) \\
& =-\frac{1}{810}\left(71 x^{2}+71 y^{2}+16 x y\right) \leqslant 0 .
\end{aligned}
$$

Also, if $x, y \in X$ such that $\alpha(x, y)=0$, then

$$
\begin{aligned}
& F(\alpha(x, y) \sigma(A x, B y), \sigma(x, y), \sigma(x, A x), \sigma(y, B y), \sigma(x, B y), \sigma(y, A x)) \\
& \quad=F(0, \sigma(x, y), \sigma(x, A x), \sigma(y, B y), \sigma(x, B y), \sigma(y, A x))=-\frac{1}{9}(\sigma(x, B y)+\sigma(y, A x)) \leqslant 0 .
\end{aligned}
$$

All hypotheses of Theorem 2.1 hold and 0 is the unique common fixed point of $A$ and $B$.

Example 2.18. Take $X=[0,2]$ endowed with the b-metric-like $\sigma(x, y)=x^{2}+y^{2}+(x-y)^{2}$. Consider the mappings $A, B: X \rightarrow X$ given by

$$
A x=\left\{\begin{array}{ll}
\frac{x^{2}}{10(1+x)}, & \text { if } x \in[0,1], \\
\frac{1}{20}+\ln x, & \text { if } x \in(1,2],
\end{array} \text { and } B x= \begin{cases}\frac{x^{2}}{10(1+x)}, & \text { if } x \in[0,1] \\
1, & \text { if } x \in(1,2] .\end{cases}\right.
$$


Define the mapping $\alpha: X \times X \rightarrow[0, \infty)$ by

$$
\alpha(x, y)= \begin{cases}1, & \text { if } x, y \in[0,1] \\ 0, & \text { otherwise. }\end{cases}
$$

Note that $(X, \sigma)$ is a complete b-metric-like space. Also, $(A, B)$ is an $\alpha$-admissible pair. In fact, let $x, y \in X$ such that $\alpha(x, y) \geqslant 1$, so $x, y \in[0,1]$. In this case

$$
\alpha(A x, B y)=\alpha\left(\frac{x^{2}}{10(1+x)}, \frac{y^{2}}{10(1+y)}\right)=1 \quad \text { and } \quad \alpha(B y, A x)=\alpha\left(\frac{y^{2}}{10(1+y)}, \frac{x^{2}}{10(1+x)}\right)=1 .
$$

Then $(A, B)$ is an $\alpha$-admissible pair.

Now, we shall show that $(A, B)$ is an $\alpha$-implicit contractive pair. Let $x, y \in X$ such that $\alpha(x, y)=1$. So $x, y \in[0,1]$. We have

$$
\begin{aligned}
\alpha(x, y) \sigma(A x, B y)=\sigma(A x, B y) & =(A x)^{2}+(B y)^{2}+(A x-B y)^{2} \\
& \left.=\left(\frac{x^{2}}{10(1+x)}\right)^{2}+\left(\frac{y^{2}}{10(1+y)}\right)^{2}+\left(\frac{x^{2}}{10(1+x)}-\frac{y^{2}}{10(1+y)}\right)\right)^{2} \\
& \leqslant\left(\frac{1}{20}\right)^{2}\left(x^{2}+y^{2}\right)+\left(\frac{9}{100}\right)^{2}(x-y)^{2} \\
& \leqslant\left(\frac{9}{100}\right)^{2} \sigma(x, y) .
\end{aligned}
$$

Also, if $x, y \in X$ such that $\alpha(x, y)=0$, then

$$
\alpha(x, y) \sigma(A x, B y)=0 \leqslant\left(\frac{9}{100}\right)^{2} \sigma(x, y) .
$$

Now, we show that $(H)$ is verified. Let $\left\{x_{n}\right\}$ be a sequence in $X$ such that $\alpha\left(x_{n}, x_{n+1}\right) \geqslant 1, \alpha\left(x_{n+1}, x_{n+}\right) \geqslant 1$ for all $n$ and $x_{n} \rightarrow u \in(X, \sigma)$ as $n \rightarrow \infty$. Then, $\left\{x_{n}\right\} \subset[0,1]$ and it is easy to see that $x_{n} \rightarrow u \in(X,||$.$) as$ $n \rightarrow \infty$. Consequently, $u \in[0,1]$ and so $\alpha\left(x_{n}, u\right)=\alpha\left(u, x_{n}\right)=1$ for all $n$. Thus, all hypotheses of corollary 2.11 are verified and 0 is the unique common fixed point of $A$ and $B$.

Example 2.19. Let $X=\{0,1,2\}$ and $\sigma: X \times X \rightarrow[0, \infty)$ be defined by

$$
\sigma(0,0)=0, \sigma(1,1)=4, \sigma(2,2)=64, \sigma(0,1)=\sigma(1,0)=1, \sigma(0,2)=\sigma(2,0)=16 \text { and } \sigma(1,2)=\sigma(2,1)=25 .
$$

Then, $(X, \sigma)$ is a complete $b$-metric-like space with coefficient $s=2$, but neither a b-metric, nor a metriclike since $\sigma(1,2)=25>17=\sigma(1,0)+\sigma(0,2)$.

Define $A, B: X \rightarrow X$ such that

$$
\mathrm{A} 0=0, \mathrm{~A} 1=\mathrm{A} 2=1, \mathrm{~B} 0=\mathrm{B} 1=0, \text { and } \mathrm{B} 2=1 .
$$

Take $\alpha: X \times X \rightarrow[0, \infty)$ defined by

$$
\left\{\begin{array}{l}
\alpha(0,0)=\alpha(0,1)=1, \\
0 \quad \text { otherwise }
\end{array}\right.
$$

Note that $(A, B)$ is an $\alpha$-admissible pair. Indeed, let $x, y \in X$ such that $\alpha(x, y) \geqslant 1$, then $(x=y=0)$ or $(x=0, y=1)$. It follows that

$$
\alpha(\mathrm{A} 0, \mathrm{~B} 0)=\alpha(\mathrm{B} 0, \mathrm{~A} 0)=\alpha(0,0)=1 \text { and } \alpha(\mathrm{A} 0, \mathrm{~B} 1)=\alpha(\mathrm{B} 1, \mathrm{~A} 0)=\alpha(0,0)=1 .
$$

Now, we shall show that the contraction condition (2.13) of Corollary 2.4 is verified for all $k \in\left[0, \frac{1}{8}\right)$. Let $x, y \in X$ such that $\alpha(x, y)=1$, then $(x=y=0)$ or $(x=0, y=1)$. We have

$$
\alpha(0,0) \sigma(A 0, B 0)=\sigma(A 0, B 0)=\sigma(0,0)=0 \leqslant k \sigma(0,0) .
$$


Also,

$$
\alpha(0,1) \sigma(\mathrm{A} 0, \mathrm{~B} 1)=\sigma(0,0)=0 \leqslant k \sigma(0,1) .
$$

Moreover, for all $x, y \in X$ such that $\alpha(x, y)=0$, we have

$$
\alpha(x, y) \sigma(A x, B y)=0 \leqslant k \sigma(x, y) .
$$

The condition contraction (2.13) holds.

For $x_{0}=0$, we have $\alpha\left(A x_{0}, x_{0}\right) \geqslant 1$ and $\alpha\left(x_{0}, A x_{0}\right) \geqslant 1$. On the other hand, $A$ and $B$ are sequentially continuous on $(X, \sigma)$. In fact, if $\left\{x_{n}\right\}$ is a sequence such that $x_{n} \rightarrow x$ in $(X, \sigma)$, it is easy to see that there exists $N \in \mathbb{N}$ such that $x_{n}=x$ for all $n \geqslant N$ and so $A x_{n}=A x$ for all $n \geqslant N$. It follows that $\sigma\left(A x_{n}, A x\right)=\sigma(A x, A x)$ for all $n \geqslant N$. Thus $A x_{n} \rightarrow A x$ in $(X, \sigma)$, so $A$ is sequentially continuous on $(X, \sigma)$. Similarly, $B$ is sequentially continuous on $(X, \sigma)$. Therefore, all the hypothesis of Corollary 2.4 are satisfied. Here, 0 is the unique common fixed point of $A$ and $B$.

\section{Acknowledgment}

The authors gratefully acknowledge the support from King Abdulaziz City for Science and Technology (KACST), Kingdom of Saudi Arabia, Project Number (SG: 36-39).

\section{References}

[1] T. Abdeljawad, E. Karapınar, K. Taş, Existence and uniqueness of a common fixed point on partial metric spaces, Appl. Math. Lett., 24 (2011), 1900-1904. 1

[2] M. A. Alghamdi, N. Hussain, P. Salimi, Fixed point and coupled fixed point theorems on b-metric-like spaces, J. Inequal. Appl., 2013 (2013) 25 pages. 1

[3] M. A. Alghamdi, E. Karapinar, G- $\beta-\psi$ contractive-type mappings and related fixed point theorems, J. Inequal. Appl., 2013 (2013), 16 pages. 1

[4] M. U. Ali, T. Kamran, E. Karapınar, $(\alpha, \psi, \xi)$-contractive multivalued mappings, Fixed Point Theory Appl., 2014 (2014), 8 pages. 1

[5] A. Aliouche, V. Popa, General common fixed point theorems for occasionally weakly compatible hybrid mappings and applications, Novi Sad J. Math., 39 (2009), 89-109. 1

[6] I. Altun, F. Sola, H. Simsek, Generalized contractions on partial metric spaces, Topology Appl., 157 (2010), $2778-2785$. 1

[7] A. Amini-Harandi, Metric-like spaces, partial metric spaces and fixed points, Fixed Point Theory Appl., 2012 (2012), 10 pages. 1

[8] H. Aydi, $\alpha$-implicit contractive pair of mappings on quasi b-metric spaces and application to integral equations, Accepted in J. Nonlinear Convex Anal., (2015). 1

[9] H. Aydi, M. F. Bota, E. Karapinar, S. Moradi, A common fixed point for weak $\phi$-contractions on b-metric spaces, Fixed Point Theory, 13 (2012), 337-346. 1

[10] H. Aydi, M. Jellali, E. Karapınar, Common fixed points for generalized $\alpha$-implicit contractions in partial metric spaces: consequences and application, Rev. R. Acad. Cienc. Exactas Fs. Nat. Ser. A Math. RACSAM , 109 (2015), 367-384. 1

[11] V. Berinde, Approximating fixed points of implicit almost contractions, Hacet. J. Math. Stat., 41 (2012), 93-102.

[12] V. Berinde, F. Vetro, Common fixed points of mappings satisfying implicit contractive conditions, Fixed Point Theory Appl., 2012 (2012), 8 pages. 1

[13] C.-F. Chen, J. Dong, C.-X. Zhu, Some fixed point theorems in b-metric-like spaces, Fixed Point Theory Appl., 2015 (2015), 10 pages. 1

[14] L. B. Ćirić, A generalization of Banach's contraction principle, Proc. Amer. Math. Soc., 45 (1974), 267-273. 2

[15] L. B. Ćirić, B. Samet, H. Aydi, C. Vetro, Common fixed points of generalized contractions on partial metric spaces and an application, Appl. Math. Comput., 218 (2011), 2398-2406. 1

[16] S. Czerwik, Nonlinear set-valued contraction mappings in b-metric spaces, Atti Sem. Mat. Fis. Univ. Modena, 46 (1998), 263-276. 1

[17] R. D. Daheriya, R. Jain, M. Ughade, Some fixed point theorem for expansive type mapping in dislocated metric space, ISRN Math. Anal., 2012 (2012), 5 pages. 1

[18] R. George, R. Rajagopalan, S. Vinayagam, Cyclic contractions and fixed points in dislocated metric spaces, Int. J. Math. Anal. (Ruse), 7 (2013), 403-411. 1

[19] N. Hussain, J. R. Roshan, V. Parvaneh, Z. Kadelburg, Fixed Points of Contractive Mappings in-Metric-Like Spaces, Scientific World J., 2014 (2014), 15 pages. 1 
[20] M. Imdad, S. Kumar, M. S. Khan, Remarks on some fixed point theorems satisfying implicit relations, Dedicated to the memory of Prof. Dr. Naza Tanović-Miller. Rad. Mat., 11 (2002), 135-143. 1

[21] M. Jleli, E. Karapınar, B. Samet, Best proximity points for generalized $\alpha-\psi$-proximal contractive type mappings, J. Appl. Math., 2013 (2013), 10 pages. 1

[22] M. Jleli, E. Karapinar, B. Samet, Fixed point results for $\alpha-\psi_{\lambda}$-contractions on gauge spaces and applications, Abstr. Appl. Anal., 2013 (2013), 7 pages. 1

[23] E. Karapınar, P. Salimi, Dislocated metric space to metric spaces with some fixed point theorems, Fixed Point Theory Appl., 2013 (2013), 19 pages. 1

[24] E. Karapinar, B. Samet, Generalized $\alpha-\psi$ contractive type mappings and related fixed point theorems with applications, Abstr. Appl. Anal., 2012 (2012), 17 pages. 1

[25] S. G. Matthews, Partial metric topology, Papers on general topology and applications, Flushing, NY, (1992), Ann. New York Acad. Sci., New York Acad. Sci., New York, 728 (1994), 183-197. 1

[26] B. Mohammadi, S. Rezapour, N. Shahzad, Some results on fixed points of $\alpha-\psi$-Ciric generalized multifunctions, Fixed Point Theory Appl., 2013 (2013), 10 pages. 1

[27] A. Pansuwan, W. Sintunavarat, V. Parvaneh, Y. J. Cho, Some fixed point theorems for $(\alpha, \theta, k)$-contractive multi-valued mappings with some applications, Fixed Point Theory Appl., 2015 (2015), 11 pages. 1

[28] V. Popa, Fixed point theorems for implicit contractive mappings, Stud. Cercet. Ştiinţ. Ser. Mat. Univ. Bacău, 7 (1997), 129-133. 1

[29] B. Samet, C. Vetro, P. Vetro, Fixed point theorems for $\alpha \psi$-contractive type mappings, Nonlinear Anal., 75 (2012), 2154-2165. 1, 1.14

[30] B. Samet, C. Vetro, F. Vetro, From metric spaces to partial metric spaces, Fixed Point Theory Appl., 2013 (2013), 11 pages. 1

[31] S. L. Singh, S. Czerwik, K. Król, A. Singh, Coincidences and fixed points of hybrid contractions, Stability of functional equations and applications, Tamsui Oxf. J. Math. Sci., 24 (2008), 401-416. 1

[32] F. Vetro, S. Radenović, Nonlinear \&-quasi-contractions of iri-type in partial metric spaces, Appl. Math. Comput., 219 (2012), 1594-1600. 1

[33] C. Vetro, F. Vetro, Metric or partial metric spaces endowed with a finite number of graphs: a tool to obtain fixed point results, Topology Appl. 164 (2014), 125-137. 1

[34] O. Yamaod, W. Sintunavarat, Y. J. Cho, Common fixed point theorems for generalized cyclic contraction pairs in b-metric spaces with applications, Fixed Point Theory Appl., 2015 (2015), 18 pages. 1

[35] O. Yamaod, W. Sintunavarat, Y. J. Cho, Existence of a common solution for a system of nonlinear integral equations via fixed point methods in b-metric spaces, Open Math., 14 (2016), 128-145. 1 